\title{
Lentiviral vector-mediated doxycycline-inducible USP39 shRNA or cDNA expression in triple-negative breast cancer cells
}

\author{
SHIHAI LIU ${ }^{1}$, XIANGPING LIU ${ }^{1}$, HAIBO WANG ${ }^{2}$, QUAN ZHOU $^{1}$, YE LIANG $^{1}$, \\ AIHUA SUI $^{1}$, RUYONG YAO ${ }^{1}$, BIN ZHAO $^{2}$ and MING SUN ${ }^{2}$ \\ ${ }^{1}$ Medical Research Center and ${ }^{2}$ Center of Diagnosis and Treatment of Breast Disease, \\ The Affiliated Hospital of Qingdao University, Qingdao, Shandong 266003, P.R. China
}

Received December 29, 2014; Accepted February 24, 2015

DOI: $10.3892 /$ or.2015.3872

\begin{abstract}
Triple-negative breast cancer (TNBC), characterized by distinct biological and clinicopathological features, has a poor prognosis due to lack of effective therapeutic targets. Our previous data revealed that high levels of USP39 were selectively present in TNBC samples compared with their normal breast tissue samples and USP39 was also expressed at different levels in cultured TNBC cells and normal breast cells. Yet, the underlying cellular and molecular mechanisms of USP39 remain unclear. In the present study, we describe a doxycycline (DOX)-regulated lentiviral vector system expressing shRNA or cDNA of the USP39 gene in the TNBC cell line MDA-MB-231. USP39 expression was knocked down by the miR-30-based inducible lentiviral short hairpin RNA (shRNA) delivery system or overexpressed by the inducible cDNA system. The inducible shRNA-mediated downregulation of USP39 expression markedly reduced the proliferation and colony-forming ability of MDA-MB-231 cells, while overexpression of USP39 by the inducible system did not promote cancer cell proliferation. The lentiviral vector-mediated Tet-on system demonstrated efficient and inducible knockdown of USP39 or overexpression of USP39 in TNBC cells, facilitating a wide variety of applications for gene knockdown and overexpression experiments in gene functional studies in vitro and in vivo.
\end{abstract}

\section{Introduction}

Breast cancer is the most common malignancy and the leading cause of cancer-related mortality among women worldwide (1). In patients with breast cancer, survival rates have improved steadily over the past two decades. Death resulting from breast

Correspondence to: Professor Haibo Wang, Center of Diagnosis and Treatment of Breast Disease, The Affiliated Hospital of Qingdao University, Qingdao, Shandong 266003, P.R. China

E-mail: hbwang66@126.com

Key words: ubiquitin-specific protease, proliferation, triple-negative breast cancer cancer is due primarily to cancer cell invasion of the surrounding tissues and metastasis to distal organs followed by secondary tumor formation (2). However, triple-negative breast cancer (TNBC) exhibits aggressive characteristics associated with shorter disease-free survival (3). TNBC tumors are characterized by the absence of estrogen receptor (ER) and progesterone receptor (PR) expression as well as human epidermal growth factor receptor 2 (HER-2) amplification. Therefore, patients with TNBCs do not benefit from commonly used antiestrogen and herceptin-based therapies (4,5). Although chemotherapy is currently the mainstay of systemic treatment for breast cancer, patients with TNBC disease have a worse outcome after chemotherapy than patients with other subtypes of breast cancer $(6,7)$. Previous studies revealed that neoadjuvant treatment involving the administration of chemotherapy before surgery was effective in a minority of women with TNBCs who showed a complete pathologic response and an excellent outcome; however, a relatively poor outcome was observed for the majority of the population with residual disease after treatment.

Ubiquitin is a small regulatory protein that is found in almost all tissues of eukaryotic organisms. Ubiquitination, the covalent attachment of ubiquitin to a target protein, is a post-translational modification that regulates the stability, function and/or localization of the modified proteins $(8,9)$. USP39, also known as $65-\mathrm{kDa}$ SR-related protein of the U4/U6-U5 tri-snRNP, has been implicated in the assembly of the mature spliceosome complex (10). USP39 is also required to maintain the spindle checkpoint and to support successful cytokinesis. Consistent with its previously described role in mRNA processing, depletion of USP39 leads to a specific reduction of the mRNA levels of Aurora B (11), and mutation of zebrafish USP39 leads to rb1 splicing defect and pituitary lineage expansion (12). In addition, USP39 and USP4 can form a stable complex in the cell (13). The USP4 contains a Ubl (ubiquitin-like) domain, and this Ubl domain can bind to the catalytic domain and compete with the ubiquitin substrate, partially inhibiting USP4 activity against different substrates. Notably, USP39 relieves this inhibition (14).

In the field of mammalian cell biology, a major approach to reveal one or several gene functions by transient/stable overexpression or knockdown of the target gene, involves manipulation of the expression of genes of interest in selected cell lines, Unfortunately, for various cell biological investiga- 
tions, this approach is unsuitable when the gene expression manipulations result in cell growth/proliferation defects or unwanted cell differentiation. Therefore, researchers have adapted the tetracycline repressor protein (TetR) from the $E$. coli tetracycline resistance operon to generate extremely efficient systems for the expression of cDNAs in mammalian cells $(15,16)$. In short, TetR has been modified to either block transcription initiation by binding to the Tet-operator (TO) in the promoter region following addition of tetracycline (termed Tet-off system) or bind to the TO in the absence of tetracycline (termed Tet-on system). Given the inconvenience that the Tet-off system requires the continuous presence of tetracycline, the Tet-on system has been more extensively optimized, resulting in the development of very efficient vector systems for cDNA expression. The aim of the present study was to elucidate the role of USP39 in TNBC cells using the Tet inducible system. Using real-time PCR analysis, we found that the USP39 gene was more active in TNBC cells than in nonTNBC cells. Therefore, we investigated USP39 in TNBC cells.

\section{Materials and methods}

Tissue samples and clinical data. Informed consent was obtained for the use of 25 human triple-negative breast cancer (TNBC) tissue samples from adult patients diagnosed at the Affiliated Hospital of Qingdao University, Qingdao, China. Twenty-five normal breast tissue samples were also obtained. The present study was approved by the hospital institutional review board and written informed consent was obtained from all patients included in the present study.

Cell culture and reagents. The human embryonic kidney cell line 293FT, human breast cancer cell lines MDA-MB-231 and HCC1937, and the normal breast cell line HS578BST, were obtained from the American Type Culture Collection (Rockville, MD, USA) and cultured in Dulbecco's modified Eagle's medium (DMEM) supplemented with $10 \%$ fetal bovine serum (FBS) and $100 \mathrm{IU} / \mathrm{ml}$ penicillin/streptomycin in a $37^{\circ} \mathrm{C}$ humidified incubator with $5 \% \mathrm{CO}_{2}(17)$.

Lentiviral production and transduction. We reconstituted the Tet-on (tetracycline-regulated transgene expression) twocomponent system in a single lentiviral vector by inserting a CMV-rtTA-T2A-reporter gene element after the TREminiCMV expression cassettes based on the pLVTHM system. To develop the miR-30 hairpin-based gene knockdown vector, the miR-30 backbone sequences were further modified to incorporate the target gene. To test miR-30 hairpin-based knockdown of USP39 in a lentiviral vector, we designed miR-30-shUSP39 to the USP39 gene. The forward (miR-30-shUSP39-F) and reverse (miR-30-shUSP39-R) oligonucleotides used were 5'-TCGAGAAGGTATATTGCTGTTG ACAGTGAGCGAAAGGTTAAGGTGAGCTCATCGTAGT GAAGCCACAGATGTACGATGAGCTCACCTTAACCTT GTGCCTACTGCCTCGG-3' and 5'-AATTCCGAGGCAGT AGGCACAAGGTTAAGGTGAGCTCATCGTACATCTGT GGCTTCACTACGATGAGCTCACCTTAACCTTTCGCTC ACTGTCAACAGCAATATACCTTC-3', respectively. These sequences were synthesized, annealed into new oligonucleotides, then ligated into the pLV-tet-on-GFP vector and designated pLV-tet-on-shUSP39-GFP. The USP39 full-length fragment was inserted into the pLV-tet-on-mCherry vector to generate the pLV-tet-on-USP39-mCherry shuttle vector. Lentiviruses were generated by cotransfecting $15 \mu \mathrm{g}$ of lentiviral vector and $7.5 \mu \mathrm{g}$ of each packaging vector (coding for Gag, Pol, Tat, Rev and VSVG) into 293FT cells using the calcium phosphate method. Supernatants were collected $48 \mathrm{~h}$ after transfection, filtered through a $0.4-\mu \mathrm{m}$ membrane and used to infect the cells. The cells were infected and the percentage of $\mathrm{GFP} / \mathrm{mCherry}$-positive cells was determined to ensure that the majority of cells contained the lentiviruses (18).

$R N A$ extraction and quantitative reverse transcription-PCR. Total RNA was isolated using RNAiso (Takara ) (19). Quantitative real-time PCR was performed using the LightCycler 480 instrument (Roche) with the SYBR-Green PCR kit (Takara). The primers used were: USP39, 5'-TTTTCCTCAACCTCCACA-3' and 5'-ATTCAGTCCCA CAATACCC-3'; and GAPDH, 5'-TCATGGGTGTGAACC ATGAGAA-3' and 5'-GGCATGGACTGTGGTCATGAG-3'. The parameters for the PCR were 1 cycle at $95^{\circ} \mathrm{C}$ for $1 \mathrm{~min}$ followed by 40 cycles at $95^{\circ} \mathrm{C}$ for $5 \mathrm{sec}$ and then $60^{\circ} \mathrm{C}$ for 20 sec. Fold-changes in mRNA levels were calculated according to the $2^{-\Delta \Delta \mathrm{Ct}}$ method using GAPDH mRNA for normalization.

Protein extraction and western blot analysis. The mockinfected and infected cells in 6-well plates were washed once with phosphate-buffered saline and lysed directly in $1 \mathrm{X}$ sodium dodecyl sulfate (SDS) sample buffer. Proteins were denatured by boiling. Equivalent amounts of protein were subjected to $10 \%$ SDS-polyacrylamide gel electrophoresis and transferred to polyvinylidene fluoride membranes (Millipore). The membranes were blocked in Tris-buffered saline-Tween containing 5\% milk and probed overnight in Tris-buffered saline-Tween at $4^{\circ} \mathrm{C}$ with anti-caspase-3 $(1: 2,000)$, anti-Bcl-2 $(1: 1,000)$, anti-Bax $(1: 1,000)$ or anti-GAPDH $(1: 5,000)$. Horseradish peroxidase-conjugated goat anti-rabbit and antimouse immunoglobulin secondary antibodies were purchased from Sigma and used at a 1:5,000 dilution. Secondary antibody was detected using ECL Plus detection reagents (Amersham Biosciences) and captured with Fusion FX6 (Vilber Lourmat). The results were quantified using ImageJ software.

MTT assay. MTT assays were performed to analyze the cell proliferation as previously described (19). In brief, 5,000 cells/well were cultured in 96-well plates. Cells were incubated with $100 \mu \mathrm{l} /$ well of 3-(4,5-dimethyl-2-thiazolyl)-2,5-diphenyltetrazolium bromide (MTT, $0.5 \mathrm{mg} / \mathrm{ml}$; Sigma) for $4 \mathrm{~h}$. After removing the medium, $100 \mu \mathrm{l}$ of DMSO was added to each well, and the plates were shaken for $10 \mathrm{~min}$. The absorbance of each well was measured at $490 \mathrm{~nm}$ using a Safire II spectrophotometer reader (Tecan, Mannedorf, Switzerland).

Colony formation assay. For the colony formation assay, cells were seeded evenly in 6 -well plates $\left(2 \times 10^{2}\right.$ cells/well $)$ and cultured for 14 days. Next, the cells were fixed with methanol for $10 \mathrm{~min}$, stained with $0.1 \%$ crystal violet for $20 \mathrm{~min}$, and washed 3 times with phosphate-buffered saline. Each treatment group was assayed in triplicate. 
A

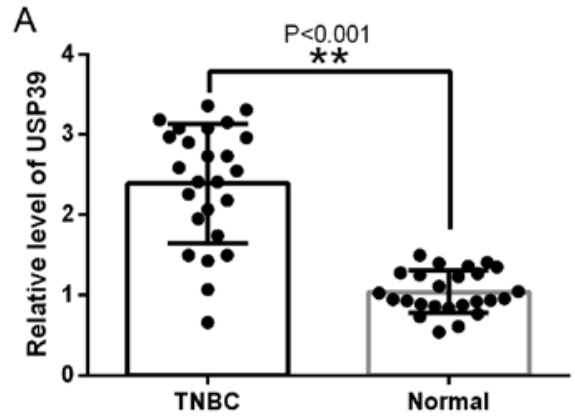

C

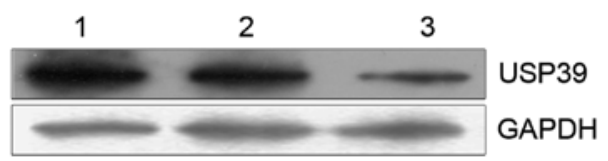

B

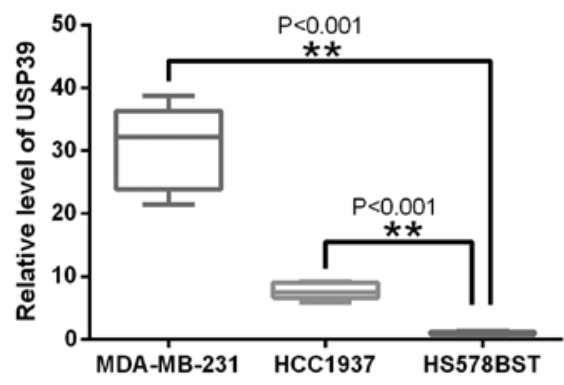

D

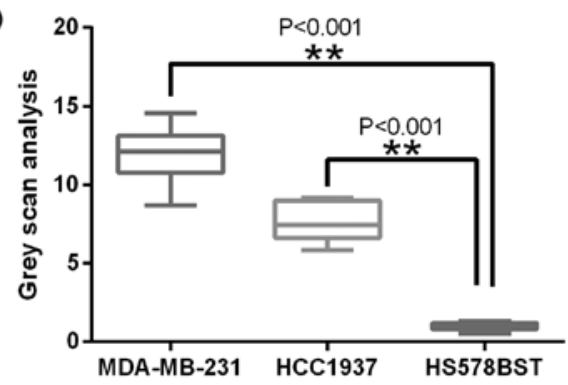

Figure 1. USP39 is highly expressed in TNBC samples. (A) USP39 expression in different human breast samples by $q-R T-P C R ; ~ * * 2<0.001$. (B) qRT-PCR analysis indicates that MDA-MB-231 and HCC1937 cells expressed high levels of USP39 compared with the HS578BST cells. Data are presented as means $\left( \pm\right.$ SD) of six replicates; ${ }^{* *} \mathrm{P}<0.001$. (C) Western blot analysis of USP39 expression in three cell lines. Lane 1, MDA-MB-231 cells; lane 2, HCC1937 cells; lane 3, HS578BST cells. (D) Expression level of USP39 in three cells, normalized by GAPDH expression; ${ }^{* *} \mathrm{P}<0.001$.

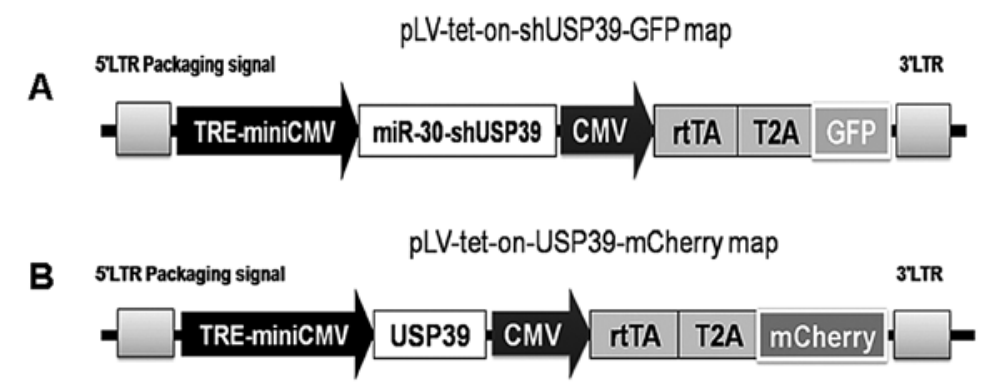

Figure 2. Schematic diagram of the self-inactivating lentiviral vectors used in the present study. (A) Schematic of the pLV-tet-on-USP39-mCherry lentiviral construct. (B) Schematic of the pLV-tet-on-shUSP39-GFP lentiviral construct.

Statistical analysis. The Student's t-test and one-way analysis of variance were used to determine significance. Error bars were used to represent the standard error of the mean. The Pearson correlation coefficient was calculated to test the association between normal and tumor samples. A P-value $<0.05$ was considered to indicate a statistically significant result.

\section{Results}

USP39 is upregulated in TNBC tissue and TNBC cancer cells. In the present study, we examined the effect of USP39 on TNBC. To evaluate USP39 expression in TNBC and normal breast tissues, RT-qPCR was used. Fig. 1A shows that USP39 was highly expressed in TNBC tissues compared with normal breast tissues $(\mathrm{P}<0.001)$. We also examined the expression levels of USP39 in TNBC cell lines (MDA-MB-231 and HCC1937) and the normal breast cell line HS578BST by RT-qPCR and western blot analysis. These cell lines displayed the same expression patterns as USP39 in the breast tissue samples (Fig. 1B-D).
Establishment of an inducible USP39-overexpression and USP39-downregulation lentiviral system. The principal aim of the present study was to determine the cellular and molecular mechanisms underlying the biological functions of USP39 in TNBC and normal breast cells. Since USP39 was normally expressed at different levels in cultured TNBC and normal breast cells (Fig. 1B-D), we engineered a tetracycline (doxycycline, DOX)-inducible lentiviral system to overexpress or downregulate USP39 (Fig. 2). To evaluate the relationship between USP39 expression and the TNBC-initiating capacity, TNBC cells (MDA-MB-231) were infected with LV-tet-onUSP39-mCherry or LV-tet-on-shUSP39-GFP (Fig. 3) in which the USP39 shRNA (Fig. 3A) or the USP39 cDNA (Fig. 3B) was cloned downstream of TRE-miniCMV. MDA-MB-231 cells infected with LV-tet-on-USP39-mCherry or LV-tet-onshUSP39-GFP were grown either in the absence (left panel) or the presence (right panel) of DOX.

In the cells transfected with these lentiviruses, USP39 expression was evaluated by real-time PCR and western blot analysis. USP39 expression was significantly elevated in the 

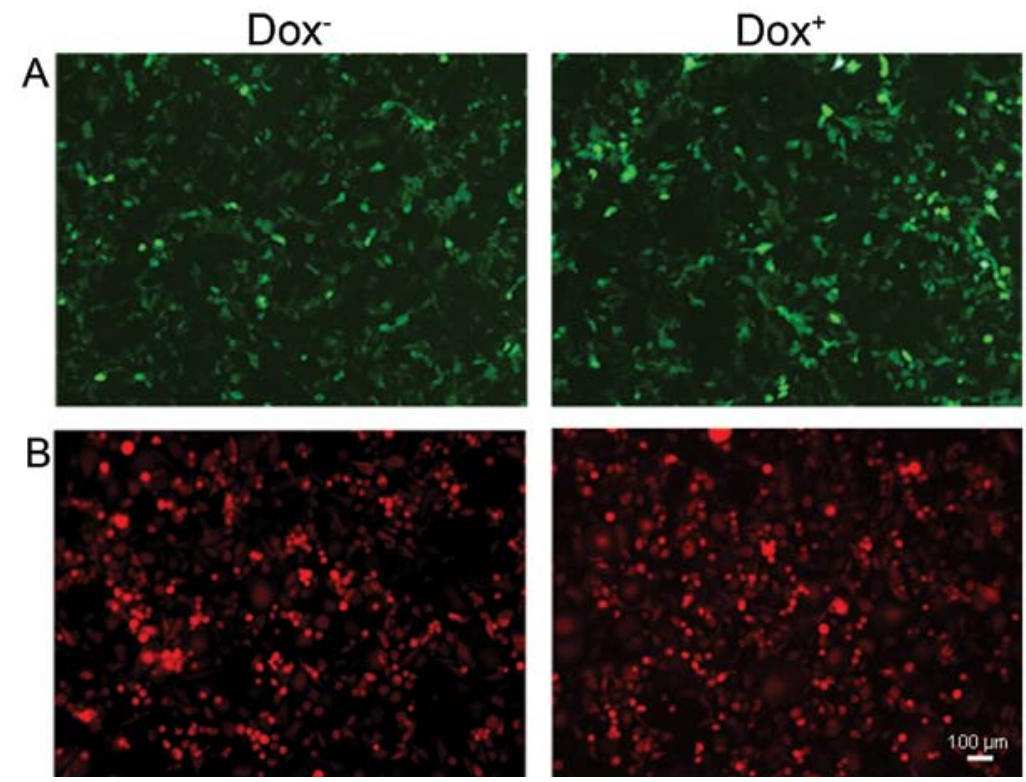

Figure 3. Establishment and characterization of a DOX-inducible USP39 overexpression and USP39 downregulation system in MDA-MB-231 cells. MDA-MB-231 cells were infected with LV-tet-on-USP39-mCherry or LV-tet-on-shUSP39-GFP and grown in T75 flasks. DOX was added to the culture medium in the DOX group. Images were obtained using a fluorescence microscope. (A) LV-tet-on-shUSP39-GFP infection. (B) LV-tet-on-USP39-mCherry infection. Scale bar, $100 \mu \mathrm{m}$.
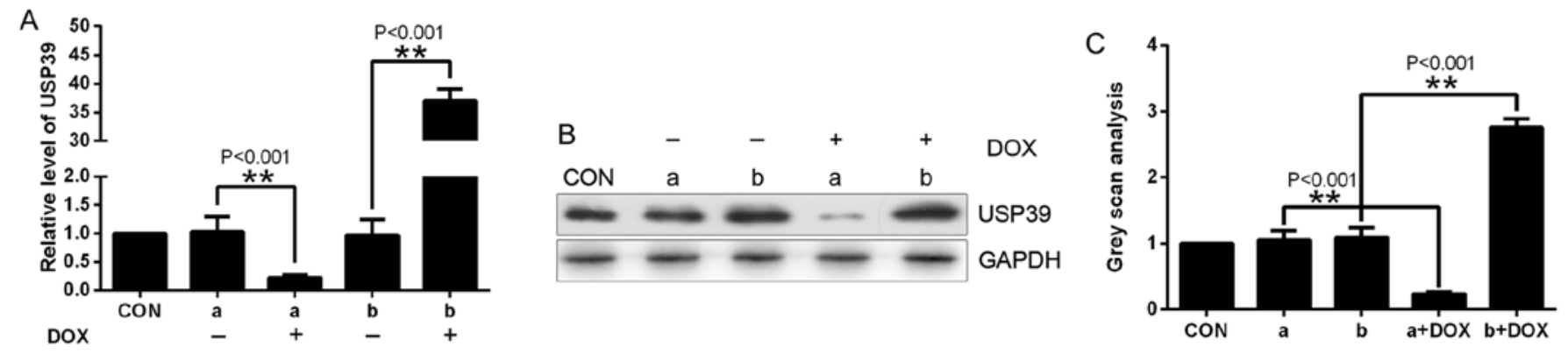

Figure 4. Real-time PCR and western blot analysis of the expression of USP39 in the different MDA-MB-231 cells. (A) Real-time PCR analysis of the expression of USP39 in the different MDA-MB-231 cells with or without DOX induction. (a) LV-tet-on-shUSP39-GFP lentivirus infection. (b) LV-tet-on-USP39-mCherry lentivirus infection. (B) Western blot analysis of USP39 in extracts derived from control MDA-MB-231, LV-tet-on-shUSP39-GFP or LV-tet-on-USP39mCherry, either untreated or induced with DOX (48 h). (a) LV-tet-on-shUSP39-GFP lentivirus infection. (b) LV-tet-on-USP39-mCherry lentivirus infection. (C) Relative expression of USP39, as evaluated by gray scan analysis. (CON) Control MDA-MB-231 cells. (a) MDA-MB-231 cells infected with LV-tet-onshUSP39-GFP. (b) MDA-MB-231 cells infected with LV-tet-on-USP39-mCherry. (a+DOX) MDA-MB-231 cells infected with LV-tet-on-shUSP39-GFP and cultured in the presence of DOX. (b+DOX) MDA-MB-231 cells infected with LV-tet-on-USP39-mCherry and cultured in the presence of DOX. Data shown are the mean $\pm \mathrm{SD}$ of a representative experiment performed in triplicate; ${ }^{* *} \mathrm{P}<0.001$

mCherry-positive cells treated with DOX compared with the control cells (Fig. 4), and USP39 was much lower in the GFP-positive cells treated with DOX compared with the control cells, as assessed using real-time PCR (Fig. 4A) and western blot analysis (Fig. 4B and C).

USP39 overexpression does not promote cancer cell proliferation, while downregulation of USP39 suppresses the growth of TNBC cells. Having successfully established an inducible system to overexpress or downregulate USP39, we next sought to determine the biological responses of TBNC cells to elevated USP39 protein. As the downregulation of USP39 was previously reported to suppress the growth of breast cancer cells in our laboratory (18), we first performed short-term assays in which our inducible-USP39 cell lines were stimulated with DOX prior to counting the total number of cells.
For functional live cell staining, it is important to verify that, after MTT formazan staining, cells can still maintain their biological functions and/or form characteristic cell aggregates. Therefore, we investigated the feasibility of staining cells with MTT formazan multiple times to detect cell growth. In parallel experimental groups, MDA-MB-231 cells without DOX induction were incubated with MTT from 24 to $72 \mathrm{~h}$. At specific time-points, the cells were washed with PBS, stained with MTT and subjected to OD490 detection. Under standard culture conditions, a significant difference was apparent in the MDA-MB-231 cells containing LV-tet-onshUSP39-GFP in the presence of DOX (Fig. 5). However, no significant differences were detected between the $\mathrm{CON}$ and LV-tet-on-USP39-mCherry groups (Fig. 5). Colony formation assays revealed differences between CON and LV-tet-onshUSP39-GFP, which suggested that decreased USP39 


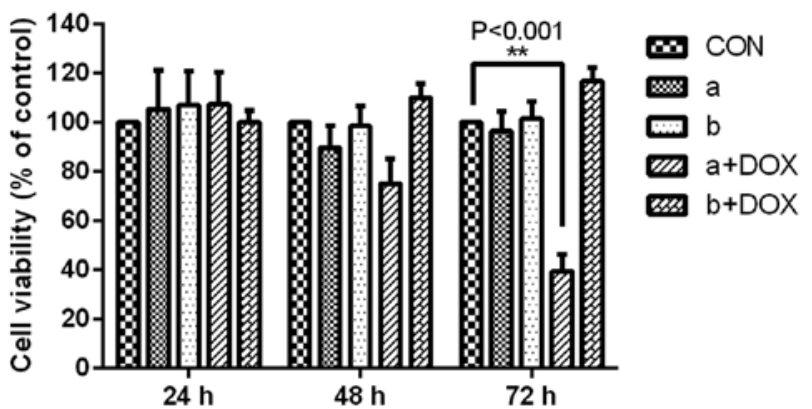

Figure 5. Determination of cell growth by MTT assays. The MDA-MB-231 cell growth rate and the results of the relative rates of cell growth were applied to absorbance at $490 \mathrm{~nm}$, relative to the MDA-MB-231 control cells (CON). The viability of MDA-MB-231 cells infected with LV-tet-on-shUSP39-GFP plus DOX was significantly decreased, and the highest inhibitory rate was $60.47 \pm 6.91 \%$ at $72 \mathrm{~h}$. (CON) Control MDA-MB-231 cells. (a) MDA-MB-231 cells infected with LV-tet-on-shUSP39-GFP. (b) MDA-MB-231 cells infected with LV-tet-on-USP39-mCherry. (a+DOX) MDA-MB-231 cells infected with LV-tet-on-shUSP39-GFP and cultured in the presence of DOX. (b+DOX) MDA-MB-231 cells infected with LV-tet-on-USP39-mCherry and cultured in the presence of DOX. Data represent the mean $\pm \mathrm{SD}$ of a representative experiment performed in triplicate; ${ }^{* * *} \mathrm{P}<0.001$.
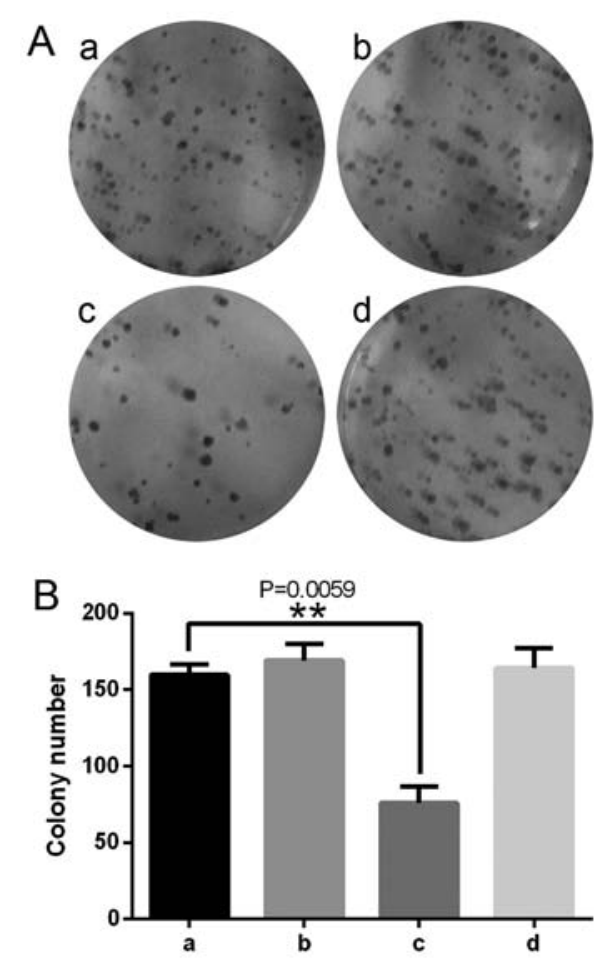

Figure 6. Cell growth evaluated by colony formation assay. MDA-MB-231 cells were examined by culturing tumor cells in $60-\mathrm{mm}$ Petri dishes for 14 days. (A) The number of colonies in each assay was quantified using the FX6 imaging system, and colony formation of tumor cells infected with different lentiviruses was assessed. (a) MDA-MB-231 cells infected with LV-tet-on-shUSP39-GFP. (b) MDA-MB-231 cells infected with LV-tet-on-USP39-mCherry. (c) MDA-MB-231 cells infected with LV-tet-onshUSP39-GFP and cultured in the presence of DOX. (d) MDA-MB-231 cells infected with LV-tet-on-USP39-mCherry and cultured in the presence of DOX. (B) Quantitative results of the colony formation was analyzed by infection with different lentiviruses. Mean values and standard deviations from the colony numbers determined in at least four wells were counted. (a) MDAMB-231 cells infected with LV-tet-on-shUSP39-GFP. (b) MDA-MB-231 cells infected with LV-tet-on-USP39-mCherry. (c) MDA-MB-231 cells infected with LV-tet-on-shUSP39-GFP and cultured in the presence of DOX. (d) MDA-MB-231 cells infected with LV-tet-on-USP39-mCherry and cultured in the presence of DOX. ** Significant inhibition $(\mathrm{P}<0.01)$ of tumor cell colony formation in the different MDA-MB-231 tumor cells.

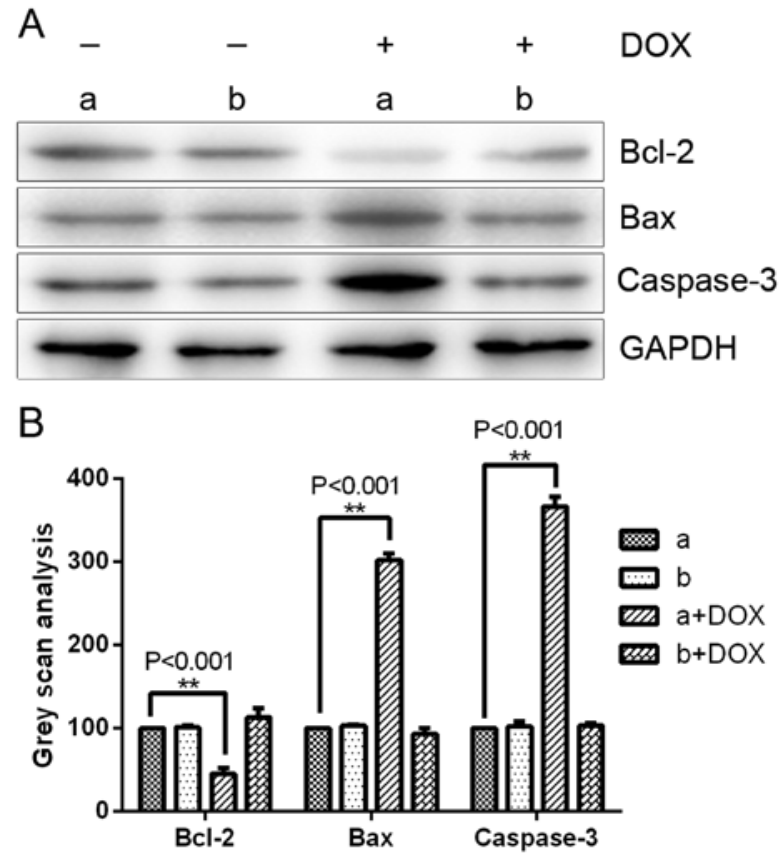

Figure 7. (A) Western blot analysis of Bcl-2, Bax and caspase- 3 in the MDA-MB-231 cells. GAPDH served as a loading control. (a) MDA-MB-231 cells infected with LV-tet-on-shUSP39-GFP. (b) MDA-MB-231 cells infected with LV-tet-on-USP39-mCherry. (B) Relative expression of USP39, as evaluated by gray scan analysis. (a) MDA-MB-231 cells infected with LV-tet-on-shUSP39-GFP. (b) MDA-MB-231 cells infected with LV-tet-on-USP39-mCherry. (a+DOX) MDA-MB-231 cells infected with LV-tet-on-shUSP39-GFP and cultured in the presence of DOX. (b+DOX) MDA-MB-231 cells infected with LV-tet-on-USP39-mCherry and cultured in the presence of DOX. Data represent the mean \pm SD of a representative experiment performed in triplicate; ${ }^{* *} \mathrm{P}<0.001$.

expression inhibited MDA-MB-231 cell growth in the TNBC cancer cells (Fig. 6).

Consistent with the observed phenotype, western blot analysis of DOX-treated MDA-MB-231 cells revealed that with the clear downregulation of USP39 protein, there was a detectable change in the levels of several regulators of cell apoptosis, including Bax and caspase-3, both of which have been previously reportedly to be transcriptionally upregulated in response to apoptosis in several types of human cancer cells $(20,21)$. Similar to these cell apoptosis regulators, the apoptosis-related gene Bcl-2 also showed significant downregulation following DOX induction in the LV-tet-on-shUSP39-GFP infection groups (Fig. 7). In contrast, MDA-MB-231 cells infected with LV-tet-on-USP39-mCherry with DOX induction, compared to the control cells without DOX induction showed no significant changes in the MTT, colony formation or western blot assays (Figs. 5-7).

\section{Discussion}

The treatment of patients with triple-negative breast cancer (TNBC), which lacks estrogen receptor (ER) and progesterone receptor (PR) expression as well as human epidermal growth factor receptor 2 (HER2) amplification, is challenging due to the heterogeneity of the disease and the absence of well-defined molecular targets $(22,23)$. TNBC tumors are generally larger in size, are of higher grade with other breast cancers, have 
lymph node involvement at diagnosis and are biologically more aggressive (24).

Recently, miRNA-based lentiviral vectors have been used for the expression of shRNAs (25-27). The shRNA embedded in the microRNA scaffold provides more robust expression of the siRNA and gene silencing compared with conventional shRNA constructs (28). Pol II-driven polycistronic transcripts containing multiple shRNA sequences can be efficiently expressed from the miRNA backbone (29). The present observations are consistent with the recent discovery that endogenous shRNA expression is enhanced by miRNAs $(30,31)$. However, it is important to note that conventional stem-loop shRNAs, such as those used previously in lentiviruses (18), are not processed into functional siRNAs under certain conditions. The inducible expression of transgenes provides an improved level of safety since it avoids much of the unintended consequences of viral vector-mediated delivery and gene silencing. We believe that Tet-on systems greatly facilitate the analysis of gene function, particularly in cell systems that are difficult to manipulate and/ or when the manipulated gene is essential for cell survival. Furthermore, the ability to control gene expression with highly specific Tet-on systems offers the opportunity to study gene functions at different stages (for example during cell cycle progression or well-defined differentiation processes). The lentiviral-mediated inducible expression of USP39-shRNA, under the control of the hU6 promoter, decreased the growth of MDA-MB-231 cancer cells. Our data provide evidence that the lentiviral-mediated Tet-on inducible system may have viable therapeutic application. Our data strongly indicate that if the expression of certain genes can be controlled by drugs in various types of cancers, the lentiviral-mediated Tet-on inducible system can be utilized for therapeutic purposes to cure cancers via the induction of cancer cell-specific death. The method presented herein will enable researchers to generate the desired Tet-on cell lines within a reasonable time frame. In addition, this approach may be applicable to other therapeutic areas through the use of different therapeutic genes in vitro and even in vivo.

USP39, which harbors a Dub domain, belongs to the ubiquitin-specific protease family. Previous investigations have described the role of USP39 in mRNA processing (32). It is essential for the assembly of mature spliceosomes and mitotic spindle checkpoint integrity $(10,11)$. However, no USP39specific substrate has been identified, and other functions of USP39 remain poorly understood. We found that suppression of USP39 in TNBC cell lines was key to their capacity for growth. Transient shRNA silencing by the USP39 lentivirus in MCF-7 cells partially recapitulated the aggressive basal phenotype (18). These effects on migration were independent of cell replication.

In summary, the present study demonstrated that the elements needed for DOX-regulated gene expression were delivered using lentiviral vectors. This system may be useful for long-term gene therapy applications and for the functional identification of genes.

\section{Acknowledgements}

The present study was supported by grants from the National Natural Science Foundations of China (81372632/H1617), the
Natural Science Foundation of Shandong Province of China (Y2008C48) and the Department of Education of Shandong Province of China (J11LF05).

\section{References}

1. Shin HR, Carlos MC and Varghese C: Cancer control in the Asia Pacific region: current status and concerns. Jpn J Clin Oncol 42: 867-881, 2012.

2. Jemal A, Bray F, Center MM, et al: Global cancer statistics. CA Cancer J Clin 61: 69-90, 2011.

3. Foulkes WD, Smith IE and Reis-Filho JS: Triple-negative breast cancer. N Engl J Med 363: 1938-1948, 2010.

4. Ferguson LL, Curran B, Martinez M, et al: Triple-negative breast cancer: what is known about it? Clin J Oncol Nurs 18: E6-E11, 2014.

5. Hirshfield KM and Ganesan S: Triple-negative breast cancer: molecular subtypes and targeted therapy. Curr Opin Obstet Gynecol 26: 34-40,2014.

6. Gangi A, Chung A, Mirocha J, et al: Breast-conserving therapy for triple-negative breast cancer. JAMA Surg 149: 252-258, 2014.

7. Chin YR, Yoshida T, Marusyk A, et al: Targeting Akt3 signaling in triple-negative breast cancer. Cancer Res 74: 964-973, 2014.

8. Dikic I, Wakatsuki S and Walters KJ: Ubiquitin-binding domains - from structures to functions. Nat Rev Mol Cell Biol 10: 659-671, 2009.

9. Pickart CM and Eddins MJ: Ubiquitin: structures, functions, mechanisms. Biochim Biophys Acta 1695: 55-72, 2004.

10. Makarova OV, Makarov EM and Luhrmann R: The 65 and $110 \mathrm{kDa}$ SR-related proteins of the U4/U6.U5 tri-snRNP are essential for the assembly of mature spliceosomes. EMBO J 20: 2553-2563, 2001.

11. van Leuken RJ, Luna-Vargas MP, Sixma TK, et al: Usp39 is essential for mitotic spindle checkpoint integrity and controls mRNA-levels of aurora B. Cell Cycle 7: 2710-2719, 2008.

12. Rios Y, Melmed S, Lin S, et al: Zebrafish usp39 mutation leads to rb1 mRNA splicing defect and pituitary lineage expansion. PLoS Genet 7: e1001271, 2011.

13. Song EJ, Werner SL, Neubauer J, et al: The Prp19 complex and the Usp4Sart3 deubiquitinating enzyme control reversible ubiquitination at the spliceosome. Genes Dev 24: 1434-1447, 2010.

14. Luna-Vargas MP, Faesen AC, van Dijk WJ, et al: Ubiquitinspecific protease 4 is inhibited by its ubiquitin-like domain. EMBO Rep 12: 365-372, 2011.

15. Gossen $M$ and Bujard H: Tight control of gene expression in mammalian cells by tetracycline-responsive promoters. Proc Natl Acad Sci USA 89: 5547-5551, 1992.

16. Gossen M, Freundlieb S, Bender G, et al: Transcriptional activation by tetracyclines in mammalian cells. Science 268: 1766-1769, 1995.

17. Kao J, Salari K, Bocanegra M, et al: Molecular profiling of breast cancer cell lines defines relevant tumor models and provides a resource for cancer gene discovery. PLoS One 4: e6146, 2009.

18. Wang H, Ji X, Liu X, et al: Lentivirus-mediated inhibition of USP39 suppresses the growth of breast cancer cells in vitro. Oncol Rep 30: 2871-2877, 2013.

19. Wang H, Zhao G, Liu X, et al: Silencing of RhoA and RhoC expression by RNA interference suppresses human colorectal carcinoma growth in vivo. J Exp Clin Cancer Res 29: 123, 2010.

20. Butt AJ, Dickson KA, McDougall F, et al: Insulin-like growth factor-binding protein-5 inhibits the growth of human breast cancer cells in vitro and in vivo. J Biol Chem 278: 29676-29685, 2003.

21. McIlroy D, Sakahira H, Talanian RV, et al: Involvement of caspase 3-activated DNase in internucleosomal DNA cleavage induced by diverse apoptotic stimuli. Oncogene 18: 4401-4408, 1999.

22. Pegram MD, Lipton A, Hayes DF, et al: Phase II study of receptor-enhanced chemosensitivity using recombinant humanized anti-p185HER $2 /$ neu monoclonal antibody plus cisplatin in patients with HER2/neu-overexpressing metastatic breast cancer refractory to chemotherapy treatment. J Clin Oncol 16: 2659-2671, 1998

23. Carey LA, Dees EC, Sawyer L, et al: The triple negative paradox: primary tumor chemosensitivity of breast cancer subtypes. Clin Cancer Res 13: 2329-2334, 2007. 
24. Haffty BG, Yang Q, Reiss M, et al: Locoregional relapse and distant metastasis in conservatively managed triple negative early-stage breast cancer. J Clin Oncol 24: 5652-5657, 2006.

25. Bauer M, Kink1 N, Meixner A, et al: Prevention of interferonstimulated gene expression using microRNA-designed hairpins. Gene Ther 16: 142-147, 2009.

26. Sun BS, Dong QZ, Ye QH, et al: Lentiviral-mediated miRNA against osteopontin suppresses tumor growth and metastasis of human hepatocellular carcinoma. Hepatology 48: 1834-1842, 2008.

27. Stegmeier F, Hu G, Rickles RJ, et al: A lentiviral microRNAbased system for single-copy polymerase II-regulated RNA interference in mammalian cells. Proc Natl Acad Sci USA 102: 13212-13217, 2005.

28. Boden D, Pusch O, Silbermann R, Lee F, Tucker L and Ramratnam B: Enhanced gene silencing of HIV-1 specific siRNA using microRNA designed hairpins. Nucleic Acids Res 32: 1154-1158, 2004.
29. Snyder LL, Esser JM, Pachuk CJ, et al: Vector design for liverspecific expression of multiple interfering RNAs that target hepatitis B virus transcripts. Antiviral Res 80: 36-44, 2008.

30. Liu XY, Tang QS, Chen HC, et al: Lentiviral miR30-based RNA interference against heparanase suppresses melanoma metastasis with lower liver and lung toxicity. Int J Biol Sci 9: 564-577, 2013.

31. Lee Y, Kim M, Han J, et al: MicroRNA genes are transcribed by RNA polymerase II. EMBO J 23: 4051-4060, 2004.

32. Lygerou Z, Christophides G and Seraphin B: A novel genetic screen for snRNP assembly factors in yeast identifies a conserved protein, Sad1p, also required for pre-mRNA splicing. Mol Cell Biol 19: 2008-2020, 1999. 